\title{
Zero sound in triplet-correlated superfluid neutron matter
}

\author{
L. B. Leinson
}

\begin{abstract}
The linear response of a superfluid neutron liquid onto external vector field is studied for the case of ${ }^{3} P_{2}-{ }^{3} F_{2}$ pairing. In particular, we analyze the case of neutron condensation into the state with $m_{j}=0$ which is conventionally considered as the preferable one in the bulk matter of neutron stars. Consideration is limited to the case when the wave-length of a perturbation is large as compared to the coherence length in the superfluid matter and the transferred energy is small in comparison with the gap amplitude. The obtained results are used to analyse collisionless soundlike excitations of the superfluid condensate. Zero sound (if it exists) is found to be anisotropic and undergoes strong decrement below some temperature threshold depending substantially on the intensity of Fermi-liquid interactions.
\end{abstract}




\section{INTRODUCTION}

At temperatures below the critical value $T_{c}$ the neutron Fermi liquid in the bulk matter of neutron stars is expected to develop a triplet superfluid condensate [1, 2]. Considerable work has done with the most realistic nuclear potentials for determining the magnitude of the superfluid gap at different matter densities [3-8], while the low-energy collective excitations of such superfluid liquid are not well investigated as yet. In the meantime such excitations can play an important role in the evolution of neutron stars. For example, the decay of collective spin waves into neutrino pairs, occuring in a superfluid core of neutron star through neutral weak currents, presents a new mechanism of intensive cooling in some domain of low temperatures [9-11]. A recent investigation [12] has shown sound-like oscillations of ${ }^{1} S_{0}$ superfluid neutron matter (called superfluid phonons) due to a very large mean-freepath influence heat conduction in a magnetized crust of neutron stars, where the motion of electrons is very anisotropic. Analogous effects might be expected in the neutron star core, since spontaneous breaking of rotation invariance and the baryon number caused by the triplet condensation should lead to the appearance of several Goldstone modes [13].

Generally speaking under $\beta$-equilibrium the superdense core of neutron stars is composed of neutrons with a small admixture of protons and electrons. A fraction of hyperons can also appear at higher densities. It is well known however that long-range electromagnetic interactions push out collective oscillations of the charged particles up to the plasma frequency which is large sufficiently for (approximate) decoupling of the plasma modes from the sound-like oscillations of neutral component [14-16]. In this case the effect of short-range proton-neutron interactions is reduced mostly to renormalization of the effective mass of neutrons participating in the collective oscillations [17, 18]. Therefore the problem can be simplified considering the sound-like excitations in a pure neutron superfluid liquid.

Previously the sound modes at finite temperatures have been investigated for isotropic singlet-spin superfluids [19] and for the case of triplet $p$-wave pairing in superfluid liquid ${ }^{3} \mathrm{He}$ [20-22]. Although a qualitative picture of the sound-like waves in superfluids is very similar, the above theories cannot be immediately applied to the case of superdense superfluid neutron matter. The well-developed theory of isotropic pairing cannot be applied because the triplet condensate in superdense nuclear matter is expected to be anisotropic. The theory of sound-like collective excitations in an anisotropic phase of superfluid ${ }^{3} \mathrm{He}$ is designed only 
for extremely fast waves with a velocity that is very large as compared to the Fermi velocity.

Since spin-orbit and tensor interactions between neutrons are known to dominate at high densities the neutron pairing involves a mixing of ${ }^{3} P_{2}$ and ${ }^{3} F_{2}$ channels [3-8]. The sum of spin-orbit and tensor interactions cannot be described with the aid of a sole coupling constant. This complicates the investigation of collective excitations in standard ways with making use of an explicit form of the pairing interaction, where the sole coupling constant drops out of the equations by virtue of the gap equation.

However, when the wave-length of the perturbation is large as compared to the coherence length in the superfluid matter and the transferred energy is small in comparison with the gap amplitude, as is typical for sound-like excitations, the collective motion of the condensate can be described in terms of total variable phase which (in the BCS approximation) can be derived immediately from the current conservation condition. This approach, for the first time suggested in Ref. [23], allows to avoid any explicit form of the interaction in the pairing channel. Residual Fermi-liquid interactions can be incorporated into the theory as a set of molecular fields [24]. In application to polarization functions, this approach is well developed in Ref. [18].

This paper is organized as follows. Section II contains some preliminary notes on how the Fermi-liquid interactions can be reduced to molecular fields. In Sec. III we derive, in the BCS approximation, the linear response of the triplet-correlated superfluid neutron liquid onto an effective vector field given by the sum of external and molecular fields. In Sect. IV we express self-consistently the effective fields via external fields, thus obtaining the linear medium response by taking into account the Fermi-liquid interactions. In Sect. V we analyze the poles of the longitudinal response function in order to derive the dispersion of sound-like oscillations in the condensate. Section VI contains a short summary of our findings and the conclusion. Throughout this paper, we use the system of units $\hbar=c=1$, and the Boltzmann constant $k_{B}=1$.

\section{FERMI-LIQUID INTERACTIONS AND MOLECULAR FIELDS}

It is well known that the Landau theory of a normal Fermi-liquid is based on the fact that a large part of the interactions can be taken into account with the aid of renormalizations effects. An effective Hamiltonian of the system contains the renormalized single-particle en- 
ergy of quasiparticles with occupation numbers $n(\mathbf{p}, \sigma)$ and a residual interaction between changes $\delta n$ in the quasiparticle occupation at the Fermi surface. As has been shown by Leggett [25], the quasiparticle pairing does not change the net occupation for a given direction on the Fermi surface, if approximate particle-hole symmetry is maintained. Thus the Fermi-liquid interactions remain unchanged upon pairing. In other words, the Fermi-liquid interactions do not interfere with the pairing phenomenon.

In our analysis we shall also assume that the anisotropy of the order parameter, which takes place in the case of triplet-spin pairing, plays no significant role in the Fermi-liquid interactions. This assumption is clearly justified because the characteristic length associated with the Fermi-liquid interactions is of the order of the inverse Fermi momentum, $p_{F}^{-1}$, and hence is much smaller than any other characteristic length entering the problem. This allows us to disregard the spin-dependent part of Fermi-liquid interactions in the vector channel we shall consider.

Since we are interested in values of the neutron momenta near the Fermi surface, $\mathbf{p} \simeq$ $p_{F} \mathbf{n}$, the amplitudes of the Fermi-liquid interactions $f\left(\mathbf{n n}^{\prime}\right)$ can be expanded into Legendre polynomials. In the vector channel these interactions are spin-independent and can be completely described in terms of the infinite set of Landau parameters $F_{l}$. In practice even for a saturated nuclear matter one does not know the Landau parameters $F_{l}$ for $l \geq 2$, and in actual calculations they are frequently put equal zero. The remaining Fermi-liquid interactions can be written in the form

$$
\varrho f\left(\mathbf{n n}^{\prime}\right)=F_{0}+F_{1} \mathbf{n n}^{\prime}
$$

where $\varrho=p_{F} M^{*} / \pi^{2}$ is the density of states near the Fermi surface in the normal state, and the effective mass of a neutron quasiparticle is defined as $M^{*}=p_{F} / v_{F}$, where $v_{F}$ is the Fermi velocity.

This approach can be considered as a model of the Fermi-liquid interactions. It is known, however, that the Landau interactions with $l \geq 2$ do not modify the longitudinal response functions of normal (nonsuperfluid) Fermi liquid. For a one-component Fermi liquid this was demonstrated, for example, in Ref. [26]. The same result was obtained for a one-component ${ }^{1} S_{0}$ superfluid Fermi liquid in Ref. [27] where the effective vertices and the polarization functions (at $\omega, q v_{F} \ll \Delta$ ) have been found to depend only on $F_{0}$ and $F_{1}$.

Interactions (1) renormalize the normal energy of a quasiparticle in the weak external 
vector field $A^{\mu}=\left(A_{0}, \mathbf{A}\right)$ as

$$
\tilde{\varepsilon}(\mathbf{p}) \simeq \varepsilon(\mathbf{p})+c_{V} A_{0}-\frac{c_{V}}{M} \mathbf{p} \mathbf{A}+\frac{1}{\varrho} F_{0} \sum_{p, \sigma} \delta n\left(\mathbf{p}^{\prime}, \sigma^{\prime}\right)+\frac{1}{\varrho p_{F}^{2}} F_{1} \mathbf{p} \sum_{p^{\prime}, \sigma} \mathbf{p}^{\prime} \delta n\left(\mathbf{p}^{\prime}, \sigma\right) .
$$

We denote as $\varepsilon_{\mathbf{p}}=v_{F}\left(p-p_{F}\right)$ the quasiparticle energy related to the Fermi energy in the normal state; $c_{V}$ is the coupling constant, which depends on the nature of the external field, and $n\left(\mathbf{p}^{\prime}, \sigma^{\prime}\right)$ is the distribution function of neutron quasiparticles with momenta $\mathbf{p}$ and spin $\sigma$.

From Eq. (2) it is clearly seen that Fermi-liquid interactions can be reduced to molecular fields [24], defined as

$$
A_{0}^{m o l} \equiv \frac{1}{c_{V} \varrho} F_{0} \sum_{p, \sigma} \delta n(\mathbf{p}, \sigma)
$$

and

$$
\mathbf{A}^{m o l} \equiv-\frac{F_{1} M}{c_{V} \varrho p_{F}^{2}} \sum_{\mathbf{p}, \sigma} \mathbf{p} \delta n(\mathbf{p}, \sigma) .
$$

Then Eq. (2) can be reduced to the form

$$
\tilde{\varepsilon}(\mathbf{p})=\varepsilon(\mathbf{p})+c_{V}\left(A_{0}^{e f f}-\frac{\mathbf{p}}{M^{*}} \mathbf{A}^{e f f}\right),
$$

where the effective fields are given by the sum of external and molecular fields,

$$
A_{0}^{e f f}=A_{0}+A_{0}^{m o l}, \mathbf{A}^{e f f}=\frac{M^{*}}{M}\left(\mathbf{A}+\mathbf{A}^{m o l}\right) .
$$

The molecular fields depend on the charge perturbation and current density and should be calculated consistently. Therefore first we shall perform the calculation of the medium response onto the effective field and next find the explicit form of the effective fields in a self-consistent way.

\section{BCS RESPONSE IN THE LIMIT OF $\omega, q v_{F} \ll \Delta$}

The triplet order parameter in the neutron superfluid is a symmetric matrix in spin space which can be written as

$$
\hat{\mathcal{D}}(\mathbf{n})=\Delta \overline{\mathbf{b}}(\mathbf{n}) \hat{\boldsymbol{\sigma}} \hat{g},
$$

where $\hat{\boldsymbol{\sigma}}=\left(\hat{\sigma}_{1}, \hat{\sigma}_{2}, \hat{\sigma}_{3}\right)$ are Pauli spin matrices; $\hat{g} \equiv i \hat{\sigma}_{2}$, with $\hat{g} \hat{g}=-\hat{1}$; and $\hat{1}$ is the $2 \times 2$ unit matrix in spin space. In the ground state, the gap amplitude $\Delta$ is a constant 
(on the Fermi surface), and $\overline{\mathbf{b}}(\mathbf{n})$ is a real vector in spin space which we normalize by the condition $\left\langle\bar{b}^{2}(\mathbf{n})\right\rangle=1$. Hereafter the angle brackets denote angle averages, $\langle\ldots\rangle \equiv$ $(4 \pi)^{-1} \int d \mathbf{n} \ldots$. The angular dependence of the order parameter is represented by the unit vector $\mathbf{n}=\mathbf{p} / p$ which defines the polar angles $(\theta, \varphi)$ on the Fermi surface. In the components, $n_{1}=\sin \theta \cos \varphi, n_{2}=\sin \theta \sin \varphi, n_{3}=\cos \theta$.

Making use of the adopted graphical notation for the ordinary and anomalous propagators, $\hat{G}=\longrightarrow, \hat{G}^{-}(p)=\longleftarrow, \hat{F}^{(1)}=\longrightarrow$, and $\hat{F}^{(2)}=\longleftrightarrow$, it is convenient to employ the Matsubara calculation technique for the system in thermal equilibrium. Then the analytic form of the propagators is as follows (see, e.g., Ref. [9])

$$
\begin{aligned}
& \hat{G}\left(\eta_{n}, \mathbf{p}\right)=G\left(\eta_{n}, \mathbf{p}\right) \delta_{\alpha \beta}, \quad \hat{G}^{-}\left(\eta_{n}, \mathbf{p}\right)=G^{-}\left(\eta_{n}, \mathbf{p}\right) \delta_{\alpha \beta}, \\
& \hat{F}^{(1)}\left(\eta_{n}, \mathbf{p}\right)=F\left(\eta_{n}, \mathbf{p}\right) \overline{\mathbf{b}} \hat{\boldsymbol{\sigma}} \hat{g}, \quad \hat{F}^{(2)}\left(\eta_{n}, \mathbf{p}\right)=F\left(\eta_{n}, \mathbf{p}\right) \hat{g} \hat{\boldsymbol{\sigma}} \overline{\mathbf{b}},
\end{aligned}
$$

where the scalar Green's functions are of the form $G^{-}\left(\eta_{n}, \mathbf{p}\right)=G\left(-\eta_{n},-\mathbf{p}\right)$ and

$$
G\left(\eta_{n}, \mathbf{p}\right)=\frac{-i \eta_{n}-\varepsilon_{\mathbf{p}}}{\eta_{n}^{2}+E_{\mathbf{p}}^{2}}, F\left(\eta_{n}, \mathbf{p}\right)=\frac{\Delta}{\eta_{n}^{2}+E_{\mathbf{p}}^{2}} .
$$

In the above, $\eta_{n} \equiv \pi(2 n+1) T$ with $n=0, \pm 1, \pm 2, \ldots$ is Matsubara's fermion frequency, and we assume the "unitary gap matrix" in the ground state, $\hat{\mathcal{D}}(\mathbf{n}) \hat{\mathcal{D}}^{\dagger}(\mathbf{n}) \propto \hat{1}$, thus obtaining the energy of a one-particle excitation in the form $E_{\mathbf{p}}^{2}=\varepsilon_{\mathbf{p}}^{2}+\Delta^{2} \bar{b}^{2}(\mathbf{n})$, where the (temperaturedependent) energy gap, $\Delta_{\mathbf{n}}=\Delta(T) \bar{b}(\mathbf{n})$, is anisotropic.

The following notation will be used below. We designate as $L_{X, X}(\omega, \mathbf{q} ; \mathbf{p})$ the analytical continuation of the Matsubara sums:

$$
L_{X X^{\prime}}\left(\omega_{m}, \mathbf{p}+\frac{\mathbf{q}}{2} ; \mathbf{p}-\frac{\mathbf{q}}{2}\right)=T \sum_{n} X\left(\eta_{n}+\omega_{m}, \mathbf{p}+\frac{\mathbf{q}}{2}\right) X^{\prime}\left(\eta_{n}, \mathbf{p}-\frac{\mathbf{q}}{2}\right)
$$

where $X, X^{\prime} \in G, F, G^{-}$, and $\omega_{m}=2 \pi T m$ with $m=0, \pm 1, \pm 2 \ldots$

It is convenient to divide the integration over the momentum space into an integration over the solid angle $d \mathbf{n}$ and over the energy $d \varepsilon_{\mathbf{p}}$ and operate with integrals

$$
\mathcal{I}_{X X^{\prime}}(\omega, \mathbf{n}, \mathbf{q} ; T) \equiv \frac{1}{2} \int_{-\infty}^{\infty} d \varepsilon_{\mathbf{p}} L_{X X^{\prime}}\left(\omega, \mathbf{p}+\frac{\mathbf{q}}{2}, \mathbf{p}-\frac{\mathbf{q}}{2}\right) .
$$

These are functions of $\omega, \mathbf{q}$ and the direction of the quasiparticle momentum $\mathbf{p}=p \mathbf{n}$. In deriving Eq. (11) integration over $d \varepsilon_{\mathbf{p}}$ is extended to $-\infty$ since the neutron matter is extremely degenerate. 
Consider the medium response onto the effective vector field (6) in the BCS approximation. In this case the ordinary three-point vector vertices of a quasiparticle and a hole are defined in accordance with Eq. (51):

$$
\gamma^{\mu}(\mathbf{p})=\left(1, \mathbf{p} / M^{*}\right), \gamma_{\mu}^{-}(\mathbf{p})=\gamma_{\mu}(-\mathbf{p})
$$

We use greek letters for Dirac indices, $\mu=0,1,2,3$.

Variation of the anomalous self-energy $\hat{\mathcal{D}}(\mathbf{n})$ in the field $A_{\text {eff }}^{\mu}$, can be described with the aid of anomalous three-point vertices $\hat{T}_{\mu}^{(1,2)}$, defined as:

$$
c_{V} \hat{T}_{\mu}^{(1)}=\delta \hat{\mathcal{D}}^{(1)} / \delta A_{e f f}^{\mu}, c_{V} \hat{T}_{\mu}^{(2)}=\delta \hat{\mathcal{D}}^{(2)} / \delta A_{e f f}^{\mu}
$$

The anomalous vertices are $2 \times 2$ matrices in spin space which, near the Fermi surface, depend on the transferred energy momentum $k^{\mu}=(\omega, \mathbf{q})$ and the direction $\mathbf{n}=\mathbf{p} / p$ of the quasiparticle velocity.

The Ward identity implies the following relations between the anomalous vertices and the order parameter in the system [14, 28] (see also Refs. [17, 23]):

$$
k^{\mu} T_{\mu}^{(1)}=2 \hat{\mathcal{D}}(\mathbf{n}), k^{\mu} T_{\mu}^{(2)}=-2 \hat{\mathcal{D}}^{\dagger}(\mathbf{n})
$$

We now restrict our consideration to the case, when the wave-length of the perturbation is large as compared to the coherence length and the transferred energy is small in comparison with the gap amplitude, $\omega, q v_{F} \ll \Delta$. The only possible collective motion of the condensate in this case is a variation of the total phase without a change of the order parameter structure. Then the Ward identity reveals that for a uniform medium the anomalous vertices can be written in the form

$$
\hat{T}_{\mu}^{(1)}=Q_{\mu}(\omega, \mathbf{q}) \overline{\mathbf{b}} \hat{\boldsymbol{\sigma}} \hat{g}, \hat{T}_{\mu}^{(2)}=-Q_{\mu}(\omega, \mathbf{q}) \hat{g} \hat{\boldsymbol{\sigma}} \overline{\mathbf{b}}
$$

where the unknown vector function $Q_{\mu}(\omega, \mathbf{q})$ satisfies the condition

$$
k^{\mu} Q_{\mu}=2 \Delta
$$

For further progress, let us consider the retarded BCS polarization tensor in the vector channel $\Pi^{\mu \nu}(\omega, \mathbf{q})$. The latter can be found using the fact that the current in the system, $j^{\mu} \equiv(\delta \rho, \mathbf{j})$ is connected to the linear correction $\delta \hat{G}$ to the Green's function of a quasiparticle 
in the effective external field $A_{\text {eff }}^{\mu}$ and can be obtained by analytic continuation of the following Matsubara sums

$$
\begin{gathered}
\delta \rho=c_{V} T \sum_{p_{m}} \int \frac{d^{3} p}{8 \pi^{3}} \operatorname{Tr}(\delta \hat{G}) \\
\mathbf{j}=\frac{c_{V}}{M^{*}} T \sum_{p_{m}} \int \frac{d^{3} p}{8 \pi^{3}} \mathbf{p} \operatorname{Tr}(\delta \hat{G})-\frac{N c_{V}^{2}}{M^{*}} \mathbf{A}^{e f f}
\end{gathered}
$$

where $N=p_{F}^{3} /\left(3 \pi^{2}\right)$ is the total number density of neutrons.

The linear correction to the Green's function of a quasiparticle caused by the external field $A_{e f f}^{\mu}$ is given by the diagrams shown in Fig. 1, and can be written analytically as

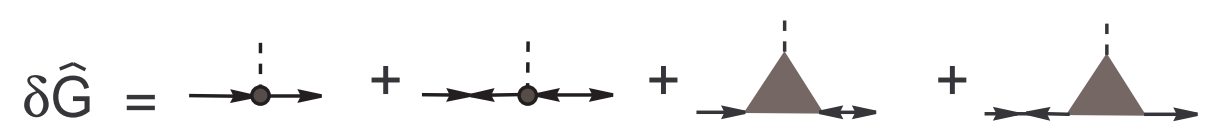

FIG. 1. Correction to the ordinary propagator of a neutron quasiparticle in the external field. The ordinary and anomalous tree-point vertices are depicted by small circles and shadowed triangles, respectively.

$$
\begin{aligned}
\delta \hat{G} & =c_{V}\left(G G \hat{\gamma}_{\mu}-F F \hat{\gamma}_{\mu}^{-}(\sigma \overline{\mathbf{b}})(\sigma \overline{\mathbf{b}})\right. \\
& \left.+G F \hat{T}_{\mu}^{(1)} \hat{g}(\sigma \overline{\mathbf{b}})+F G(\sigma \overline{\mathbf{b}}) \hat{g} \hat{T}_{\mu}^{(2)}\right) A_{e f f}^{\mu}
\end{aligned}
$$

where the anomalous vertices are to be taken in the form (15), and we use the notation $G G \equiv G\left(\eta_{n}+\omega_{m}, \mathbf{p}+\mathbf{q} / 2\right) G\left(\eta_{n}, \mathbf{p}-\mathbf{q} / 2\right)$, etc.,

Inserting Eq. (19) into Eqs. (17) and (18) we can derive the retarded polarization tensor $\Pi^{\mu \nu}$ with the aid of the standard relation $j^{\mu}=\Pi^{\mu \nu}(\omega, \mathbf{q}) A_{\nu}^{e f f}$. In this way after a little algebra we find

$$
\Pi_{\lambda \mu}=\Lambda_{\lambda \mu}+\Lambda_{\lambda} Q_{\mu}
$$

where $Q_{\mu}(\omega, \mathbf{q})$ is still an unknown vector to be found. The functions $\Lambda_{\lambda \mu}(\omega, \mathbf{q})$ and $\Lambda_{\lambda}(\omega, \mathbf{q})$ are given by

$$
\Lambda_{\lambda \mu}=c_{V}^{2} \varrho\left(\left\langle\gamma_{\lambda} \gamma_{\mu} \mathcal{I}_{G G}-\gamma_{\lambda} \gamma_{\mu}^{-} \bar{b}^{2} \mathcal{I}_{F F}\right\rangle+\frac{v_{F}^{2}}{3} \delta_{\lambda i} \delta_{i \mu}\right)
$$

and

$$
\Lambda_{\lambda}=c_{V}^{2} \varrho\left\langle 2 \gamma_{\lambda} \bar{b}^{2} \mathcal{I}_{F G}\right\rangle=c_{V}^{2} \varrho \frac{1}{\Delta}\left\langle\gamma_{\lambda}(\omega+\mathbf{q v}) \bar{b}^{2} \mathcal{I}_{F F}\right\rangle
$$


To obtain the second equality we made use of the identity

$$
\mathcal{I}_{F G}=-\mathcal{I}_{G F}=\frac{\omega+\mathbf{q v}}{2 \Delta} \mathcal{I}_{F F}
$$

which can be verified by a straightforward calculation.

The function $Q_{\mu}(\omega, \mathbf{q})$ can be found from the requirement that the polarization tensor (20) must satisfy current conservation conditions, $\Pi^{\mu \nu} k_{\nu}=0$, and $k_{\nu} \Pi^{\nu \mu}=0$, which can be written as the two coupled equations

$$
\begin{gathered}
\Lambda^{\mu \nu} k_{\nu}+2 \Delta \Lambda^{\mu}=0, \\
k_{\nu} \Lambda^{\nu \mu}+k_{\nu} \Lambda^{\nu}(q) Q^{\mu}(q)=0 .
\end{gathered}
$$

We made use of relation (16) in the first equation.

From the explicit form (21) for $\Lambda^{\mu \nu}$ one can easily find the following relation

$$
q^{\mu} \Lambda_{\mu \lambda}=\Lambda_{\lambda \mu} q^{\mu}+c_{V}^{2} \varrho\left\langle\left(\gamma_{\lambda}(\omega+\mathbf{q v})-(\omega-\mathbf{q v}) \hat{\gamma}_{\lambda}^{-}\right) \bar{b}^{2} \mathcal{I}_{F F}\right\rangle
$$

The first term on the right-hand side of this expression can be replaced as $\Lambda^{\mu \nu} k_{\nu} \rightarrow-2 \Delta \Lambda^{\mu}$, in accordance with Eq. (24). Inserting the obtained result into Eq. (25), we arrive at

$$
Q_{\lambda}=\frac{2 \Delta \Lambda_{\lambda}-c_{V}^{2} \varrho\left\langle\left(\gamma_{\lambda}(\omega+\mathbf{q v})-(\omega-\mathbf{q v}) \hat{\gamma}_{\lambda}^{-}\right) \bar{b}^{2} \mathcal{I}_{F F}\right\rangle}{q^{\mu} \Lambda_{\mu}}
$$

This formula can be simplified making use of the explicit form (22) for $\Lambda_{\lambda}$. In the components we obtain the expressions:

$$
\begin{aligned}
& Q_{0}=2 \Delta \frac{\omega\left\langle\bar{b}^{2} \mathcal{I}_{F F}\right\rangle}{\left\langle\left(\omega^{2}-(\mathbf{q v})^{2}\right) \bar{b}^{2} \mathcal{I}_{F F}\right\rangle} \\
& \mathbf{Q}=2 \Delta \frac{\left\langle\mathbf{v}(\mathbf{q v}) \bar{b}^{2} \mathcal{I}_{F F}\right\rangle}{\left\langle\left(\omega^{2}-(\mathbf{q v})^{2}\right) \bar{b}^{2} \mathcal{I}_{F F}\right\rangle},
\end{aligned}
$$

valid for $\omega, q v_{F} \ll \Delta$.

From Eqs. (20) -(22), (28) and (29) one can obtain the complete BCS polarization tensor $\Pi^{\mu \nu}(\omega, \mathbf{q})$ in the vector channel. Due to conservation of the vector current the polarization tensor can be decomposed into the sum of longitudinal (with respect to $\mathbf{q}$ ) and transverse components, where the longitudinal and transverse polarization functions are defined as $\Pi_{L} \equiv \Pi_{00}, \Pi_{T} \equiv \frac{1}{2}\left(\delta_{i j}-q_{i} q_{j} / q^{2}\right) \Pi_{i j}$, with $i, j=1,2,3$. 


\section{FERMI-LIQUID EFFECTS}

We now turn to the Fermi-liquid effects. Our aim is to express the effective fields (6) via external fields. For this we can use the fact that the density current commutes with the bare interactions and does not need to be renormalized. The current connection with the external field $\mathbf{A}$ is given by the well-known relation

$$
\sum_{p, \sigma} \mathbf{p} \delta n(\mathbf{p}, \sigma)=\frac{M}{c_{V}}\left(\mathbf{j}+\frac{N c_{V}^{2}}{M} \mathbf{A}\right) .
$$

Inserting this into Eqs. (3) and (44) we obtain the time component of the effective field (6) in the form

$$
A_{0}^{e f f} \equiv A_{0}+\frac{1}{c_{V}^{2} \varrho} F_{0} \delta \rho,
$$

Using also the continuity equation, $q j_{L}=\omega \rho$, we obtain the space components of the effective field:

$$
\begin{aligned}
& A_{L}^{e f f}=A_{L}-F_{1} \frac{M M^{*}}{\varrho p_{F}^{2} c_{V}^{2}} \frac{\omega}{q} \delta \rho \\
& \mathbf{A}_{T}^{e f f}=\mathbf{A}_{T}-F_{1} \frac{M M^{*}}{\varrho p_{F}^{2} c_{V}^{2}} \mathbf{j}_{T}
\end{aligned}
$$

Insertion of the effective fields (31) and (33) into $\delta \rho=\Pi_{L}\left(A_{0}^{\text {eff }}-(\omega / q) A_{L}^{e f f}\right)$ and $\mathbf{j}_{T}=-\Pi_{T} \mathbf{A}_{T}^{e f f}$ after a little algebra results in the following:

$$
\begin{gathered}
\delta \rho=\frac{\Pi_{L}}{1-\left(F_{0}+s^{2} F_{1} /\left(1+F_{1} / 3\right)\right) c_{V}^{-2} \varrho^{-1} \Pi_{L}}\left(A_{0}-\frac{\omega}{q} A_{L}\right), \\
\mathbf{j}_{T}=-\frac{\Pi_{T}}{1-F_{1} /\left(1+F_{1} / 3\right) v_{F}^{-2} c_{V}^{-2} \varrho^{-1} \Pi_{T}} \mathbf{A}_{T} .
\end{gathered}
$$

In obtaining this we have used the Landau formula relating the bare mass of a particle with a renormalized mass of a quasiparticle in the translation-invariant system $M^{*} / M=1+F_{1} / 3$.

The complete polarization functions $\tilde{\Pi}_{L, T}$ relate the density perturbation and the density current with the external field as $\delta \rho=\tilde{\Pi}_{L}\left(A_{0}-(\omega / q) A_{L}\right)$, and $\mathbf{j}_{T}=-\tilde{\Pi}_{T} \mathbf{A}_{T}$, respectively. Then from Eqs. (34) and (35) we obtain the complete polarization functions:

$$
\begin{gathered}
\tilde{\Pi}_{L}=\frac{\Pi_{L}}{1-\left(F_{0}+s^{2} F_{1} /\left(1+F_{1} / 3\right)\right) c_{V}^{-2} \varrho^{-1} \Pi_{L}}, \\
\tilde{\Pi}_{T}=\frac{\Pi_{T}}{1-F_{1} /\left(1+F_{1} / 3\right) v_{F}^{-2} c_{V}^{-2} \varrho^{-1} \Pi_{T}}
\end{gathered}
$$

in agreement with the results of Gusakov [18]. 
Equations (36) and (37) completely describe the non-equilibrium behavior of superfluid Fermi liquids in the vector-linear-response region for not too high $\omega$ and $q$. Explicit expressions can be written using the following notation:

$$
\begin{gathered}
\alpha(s, \mathbf{h})=\frac{1}{2}+\frac{1}{2}\left\langle\int_{0}^{\infty} d \varepsilon \frac{\left(\cos ^{2} \theta_{\mathbf{q n}}-s^{2}\right) \varepsilon^{2} / E_{\mathbf{p}}^{2}}{s^{2}-\left(\cos ^{2} \theta_{\mathbf{q n}}\right) \varepsilon^{2} / E_{\mathbf{p}}^{2}} \frac{d n}{d E_{\mathbf{p}}}\right\rangle, \\
\gamma(s, \mathbf{h})=\frac{1}{2}\left\langle\int_{0}^{\infty} d \varepsilon \frac{\cos \theta_{\mathbf{q n}}\left(\cos ^{2} \theta_{\mathbf{q n}}-s^{2}\right) \varepsilon^{2} / E_{\mathbf{p}}^{2}}{s^{2}-\left(\cos ^{2} \theta_{\mathbf{q n}}\right) \varepsilon^{2} / E_{\mathbf{p}}^{2}} \frac{d n}{d E_{\mathbf{p}}}\right\rangle, \\
\zeta(s, \mathbf{h})=\frac{1}{6}+\frac{1}{2}\left\langle\int_{0}^{\infty} d \varepsilon \frac{\cos ^{2} \theta_{\mathbf{q n}}\left(\cos ^{2} \theta_{\mathbf{q n}}-s^{2}\right) \varepsilon^{2} / E_{\mathbf{p}}^{2}}{s^{2}-\left(\cos ^{2} \theta_{\mathbf{q n}}\right) \varepsilon^{2} / E_{\mathbf{p}}^{2}} \frac{d n}{d E_{\mathbf{p}}}\right\rangle . \\
\eta(s, \mathbf{h})=\left\langle\int_{0}^{\infty} d \varepsilon \frac{\left(\cos ^{2} \theta_{\mathbf{q n}}\right) \varepsilon^{2} / E_{\mathbf{p}}^{2}}{s^{2}-\left(\cos ^{2} \theta_{\mathbf{q n}}\right) \varepsilon^{2} / E_{\mathbf{p}}^{2}} \frac{d n}{d E_{\mathbf{p}}}\right\rangle, \\
\chi(s, \mathbf{h})=\left\langle\int_{0}^{\infty} d \varepsilon \frac{s\left(\cos _{\mathbf{q n}}\right) \varepsilon^{2} / E_{\mathbf{p}}^{2}}{s^{2}-\left(\cos ^{2} \theta_{\mathbf{q n}}\right) \varepsilon^{2} / E_{\mathbf{p}}^{2}} \frac{d n}{d E_{\mathbf{p}}}\right\rangle,
\end{gathered}
$$

where $s=\omega /\left(q v_{F}\right)$,

$$
\frac{d n}{d E_{\mathbf{p}}} \equiv \frac{1}{2 T} \cosh ^{-2} \frac{E_{\mathbf{p}}}{2 T},
$$

and we assume that the angle $\theta_{\mathbf{q n}}$ between the quasiparticle momentum $\mathbf{p}=p \mathbf{n}$ and the momentum transfer $\mathbf{q}$ is fixed by the relation

$$
\cos \theta_{\mathbf{q n}}=h_{x} \sin \theta \cos \varphi+h_{y} \sin \theta \sin \varphi+h_{z} \cos \theta
$$

where the unit vector $\mathbf{h} \equiv \mathbf{q} / q=\left(h_{x}, h_{y}, h_{z}\right)$ defines the direction of the momentum transfer, and the unit vector $\mathbf{n}=(\sin \theta \cos \varphi, \sin \theta \sin \varphi, \cos \theta)$ defines the polar angles $(\theta, \varphi)$ on the Fermi surface.

Then the longitudinal polarization function (36) can be written as

$$
\tilde{\Pi}_{L}=c_{V}^{2} \varrho \frac{Q(s, \mathbf{h})}{1-\left(F_{0}+s^{2} F_{1} /\left(1+F_{1} / 3\right)\right) Q(s, \mathbf{h})},
$$

where

$$
Q(s, \mathbf{h})=\eta(s, \mathbf{h})+\chi(s, \mathbf{h})+2 \alpha(s, \mathbf{h}) \frac{s \gamma(s, \mathbf{h})+\zeta(s, \mathbf{h})}{s^{2} \alpha(s, \mathbf{h})-\zeta(s, \mathbf{h})} .
$$

\section{SOUND-LIKE EXCITATIONS}

The pole of the density fluctuation propagator (45) at

$$
\left(F_{0}+s_{0}^{2} F_{1} /\left(1+F_{1} / 3\right)\right) Q\left(s_{0}, \mathbf{h} ; T\right)=1
$$


defines the dispersion, $s=s_{0}$, of the "collisionless" collective mode, with $\omega, q v_{F} \ll \Delta$.

Equation (47) with $Q(s, \mathbf{h})$ as given in Eq. (46) generalizes previous results of Refs. [19] and [20-22] to the case of pairing caused by spin-orbit and tensor interactions. Therefore before proceeding to the detailed analysis of the sound propagation in the ${ }^{3} P_{2}-{ }^{3} F_{2}$ neutron superfluid, we examine the obtained equations for the particular cases of the triplet-spin condensate in superfluid ${ }^{3} \mathrm{He}$.

Consider first the case of isotropic pairing. If the energy gap is isotropic, the angle integrals in Eqs. (38)-(42) can be performed by assuming the polar axis along the transferred momentum. We then obtain $\gamma(s, \mathbf{h})=\chi(s, \mathbf{h})=0$ and the longitudinal polarization function reduces to Eq. (45) with

$$
Q(s)=\eta(s)+\frac{2 \alpha(s) \zeta(s)}{s^{2} \alpha(s)-\zeta(s)}
$$

as obtained by Leggett [19] for the case of isotropic $s$-wave pairing.

Consider now the case of anisotropic pairing at $T=0$. In this case the quantities $\alpha(s, \mathbf{h}) \simeq 1 / 2$ and $\zeta(s, \mathbf{h})=1 / 6$ are independent of $s$ and again isotropic, while $\gamma(s, \mathbf{h})=$ $\eta(s, \mathbf{h})=\chi(s, \mathbf{h})=0$. Thus for $T=0$ we find

$$
\tilde{\Pi}_{L}=\frac{c_{V}^{2} \varrho}{\left(1+F_{0}\right)} \frac{s_{0}^{2}}{s^{2}-s_{0}^{2}}, s_{0}^{2}=\frac{1}{3}\left(1+F_{0}\right)\left(1+F_{1} / 3\right)
$$

in agreement with the result obtained by Wölfe [21] for an anisotropic phase of superfluid ${ }^{3} \mathrm{He}$ at zero temperature. Notice that the same was obtained also by Leggett [19] for the case of isotropic $s$-wave pairing. The pole at $s=s_{0}$ corresponds to the first sound ("BogolyubovAnderson" mode) undamped at zero temperature.

We turn now to the case of anisotropic p-wave pairing in liquid ${ }^{3} \mathrm{He}$. The experimentally observable sound velocity in liquid ${ }^{3} \mathrm{He}$ is large, therefore it is traditional to calculate the sound dispersion in the limit $s \gg 1$. Expanding Eq. (46) in powers of $1 / s$, one can obtain up to accuracy $s^{-4}$,

$$
Q(s, \mathbf{h}) \simeq \frac{5}{3} \frac{1}{5 s^{2}-3}+\frac{1}{s^{4}}\left(-\left\langle\lambda(\mathbf{n}) \cos ^{4} \theta_{\mathbf{n q}}\right\rangle+\frac{\left\langle\lambda(\mathbf{n}) \cos ^{2} \theta_{\mathbf{n q}}\right\rangle^{2}}{\lambda(\mathbf{n})}\right)
$$

where

$$
\lambda(\mathbf{n}) \equiv \int_{0}^{\infty} d \varepsilon \frac{\Delta^{2}}{E^{3}} \tanh \frac{E}{2 T} .
$$


In obtaining Eq. (50) we used the identity

$$
\int_{0}^{\infty} d \varepsilon \frac{\varepsilon^{2}}{E_{\mathbf{p}}^{2}} \frac{d n}{d E_{\mathbf{p}}}=1-\int_{0}^{\infty} d \varepsilon \frac{\Delta^{2}}{E_{\mathbf{p}}^{3}} \tanh \frac{E_{\mathbf{p}}}{2 T} .
$$

Inserting Eq. (50) into Eq. (47) one can obtain the dispersion law for high-frequency sound in superfluid ${ }^{3} H e$. Assuming $F_{0} \sim s_{0}^{2} \gg 1$ we find

$$
\begin{aligned}
s_{0}^{2} & =\frac{1}{3}\left(F_{0}+\frac{9}{5}\right)\left(1+\frac{1}{3} F_{1}\right) \\
& +\left(3+F_{1}\right)\left(-\left\langle\lambda(\mathbf{n}) \cos ^{4} \theta_{\mathbf{n q}}\right\rangle+\frac{\left\langle\lambda(\mathbf{n}) \cos ^{2} \theta_{\mathbf{n q}}\right\rangle^{2}}{\lambda(\mathbf{n})}\right),
\end{aligned}
$$

in agreement with the expression derived by Wölfe [21].

We focus now on the sound propagation in the ${ }^{3} P_{2}-{ }^{3} F_{2}$ superfluid neutron liquid which is expected to exist in neutron stars at supernuclear densities. First one has to specify the order parameter (7) for the particular case of neutron pairing. It is conventional to represent the triplet order parameter of the system as a superposition of standard spin-angle functions $\hat{\Phi}_{j l m_{j}}$ of the total angular momentum $\left(j=2, m_{j}\right)$ with partial amplitudes $\Delta_{l m_{j}}$ :

$$
\hat{D}=\sum_{l m_{j}} \Delta_{l m_{j}} \hat{\Phi}_{j l m_{j}}(\mathbf{n})
$$

In our calculations we use vector notation which involves a set of mutually orthogonal complex vectors $\mathbf{b}_{l m_{j}}(\mathbf{n})$ defined as

$$
\mathbf{b}_{l m_{j}}(\mathbf{n})=-(1 / 2) \operatorname{Tr}\left(\hat{g} \hat{\boldsymbol{\sigma}} \hat{\Phi}_{j l m_{j}}\right)
$$

and normalized by the condition $\left\langle\mathbf{b}_{l^{\prime} m_{j}^{\prime}}^{*} \mathbf{b}_{l m_{j}}\right\rangle=\delta_{l l^{\prime}} \delta_{m_{j} m_{j}^{\prime}}$. We will focus on the ${ }^{3} P_{2}-{ }^{3} F_{2}$ condensation into the state with $m_{j}=0$ which is conventionally considered as the preferable one in the bulk matter of neutron stars. In this case one has

$$
\bar{b}^{2}(\mathbf{n})=\frac{1}{2}\left(1+3 n_{3}^{2}\right) \delta_{1}+\frac{3}{4}\left(5 n_{3}^{4}-2 n_{3}^{2}+1\right) \delta_{3}
$$

where $\delta_{1}=\Delta_{1}^{2} / \Delta^{2}$ and $\delta_{3}=\Delta_{3}^{2} / \Delta^{2}$ are partial contributions of the ${ }^{3} P_{2}$ and ${ }^{3} F_{2}$ states, respectively, $\delta_{1}+\delta_{3}=1$.

For $0<T<T_{c}$ the behavior of $\tilde{\Pi}_{L}$ in the intermediate region of $s$ depends essentially on the temperature. According to Eqs. (38)-(42) the imaginary part of the functions arises from the pole of the integrand at $s^{2}=\left(\cos ^{2} \theta_{\mathbf{q n}}\right) \varepsilon^{2} / E_{\mathbf{p}}^{2}$. This is Cherenkov's condition 
which can be satisfied only if $s<1$. Neglecting the narrow temperature domain where the imaginary part of polarization is exponentially small [19], one can conclude that the well-defined (undamped) waves correspond to $s>1$. Further we consider only undamped sound-like oscillations with $s>1$.

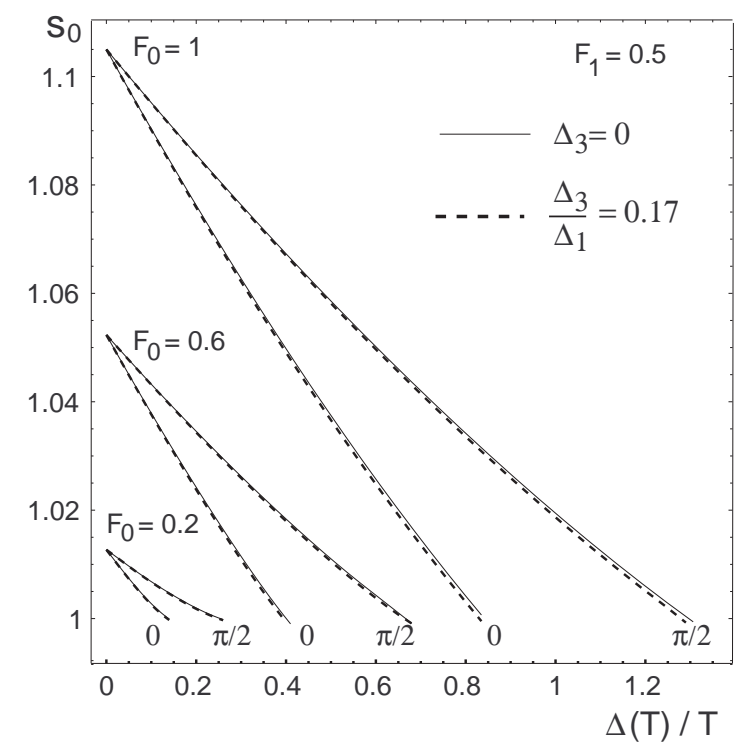

(a)

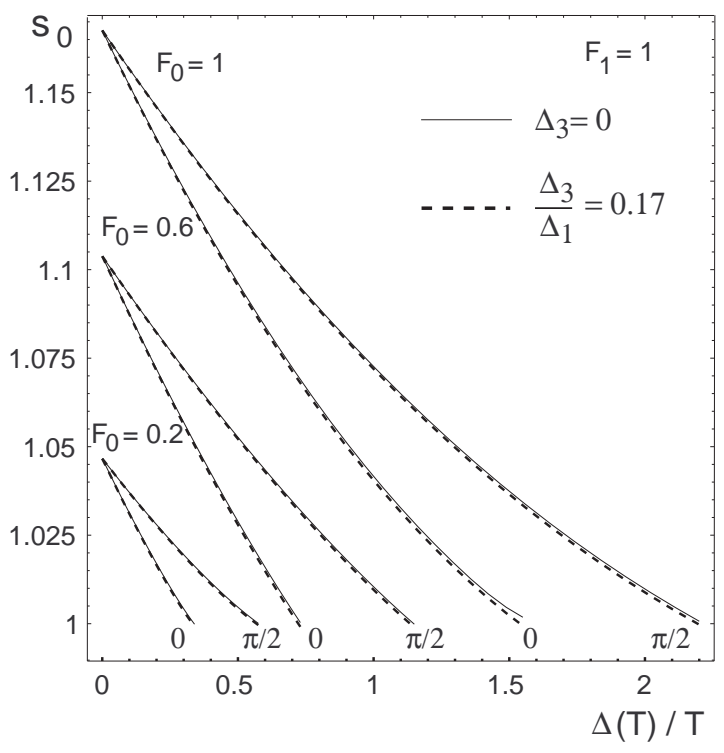

(b)

FIG. 2. Zero-sound velocity as a function of temperature below the critical point, $T<T_{c}$. The dimensionless velocity of zero sound, $s_{0}=\omega_{0} /\left(q v_{F}\right)$ is shown vs the temperature parameter $\Delta(T) / T$ for different sets of Landau parameters. Case (a): $F_{0}=0.2,0.6,1 ; F_{1}=0.5$. Case (b): $F_{0}=0.2,0.6,1 ; F_{1}=1$. We present the curves for the wave propagation along the symmetry axis $\left(\theta_{\mathbf{q}}=0\right)$ and in the perpendicular direction $\left(\theta_{\mathbf{q}}=\pi / 2\right)$.

The density-dependent Landau parameters entering the dispersion equation are not reliably known. Therefore in Fig. 2 we present solutions to Eq. (47) for several sets of the Landau parameters. The curves show the zero-sound velocity as a function of temperature parameter $\Delta(T) / T$ for a pure ${ }^{3} P_{2}$ pairing (solid curves) and for the case of pairing into the mixed ${ }^{3} P_{2}-{ }^{3} F_{2}$ state (dashed curves). The plots are made for the sound propagating along the axis of the wave function of the condensate $\left(\theta_{\mathbf{q}}=0\right)$ and in the orthogonal direction $\left(\theta_{\mathbf{q}}=\pi / 2\right)$.

For the case of mixed pairing we have chosen $\Delta_{3}=0.17 \Delta_{1}$, in agreement with that found in realistic calculations by different authors (see, e.g., Ref. [6]). As one can see, a small 
admixture of the ${ }^{3} F_{2}$ state does not modify markedly the dispersion curves obtained for the pure $p$-wave superfluid.

The sound waves are anisotropic. At fixed temperature the velocity of the sound grows along with deviation of the wave vector from the axis of the order parameter. The sound speed is maximal for orthogonal propagation.

As regards the temperature dependence, immediately below the critical temperature $T_{c}$ the velocity of zero sound goes down, and the undamped wave disappears at some temperature $T_{1}\left(\theta_{\mathbf{q}}\right)<T_{c}$ when the sound velocity becomes smaller than the Fermi velocity, $s_{0}<1$ (although the mode with exponentially small damping can exist in some region below this temperature). Thus the undamped collective excitation may or may not exist at some temperature, depending on the values of $F_{0}$ and $F_{1}$. If it does, its velocity $s_{0}\left(\theta_{\mathbf{q}}, T\right)$ will always be greater than unity.

\section{SUMMARY AND CONCLUSION}

Let us summarize our results. We have studied the linear response of a superfluid neutron liquid to an external vector field in the limit $\omega, q v_{F} \ll \Delta$. The calculation is made for the case of ${ }^{3} P_{2}-{ }^{3} F_{2}$ condensate which is expected to exist in the superdense core of neutron stars due to spin-orbit and tensor pairing interactions. By analyzing the poles of the longitudinal response we have found the low-energy spectrum of sound-like collecive excitations caused by density fluctuations in the condensate. Previously the sound-like excitations were investigated for a triplet condensate caused by central pairing forces in ${ }^{3} \mathrm{He}$ [20 22]. Our dispersion equation (47) represents a generalization of the above results to the case of pairing caused by noncentral spin-orbit and tensor interactions and naturally recovers previous results obtained for the case of central forces.

The sound-like spectrum of a Fermi liquid substantially depends on the residual particlehole interactions which are conventionally described by a set of Landau parameters. We have limited our consideration to the first two terms of this expansion. This approach can be considered as a model of the Fermi-liquid interactions, although there are indications that the higher-order Landau interactions do not affect the longitudinal response functions [26, 27].

Unfortunately, the density-dependent Landau parameters entering the dispersion equa- 
tion are not reliably known for an asymmetric nucleon matter (although, in principle, these can be evaluated theoretically [29 32]). Therefore we have studied solutions to Eq. (47) for several sets of the Landau parameters. We found that the sound waves are anisotropic and a small admixture of the ${ }^{3} F_{2}$ state does not modify markedly the dispersion curves obtained for the pure $p$-wave superfluid neutrons. At fixed temperature the velocity of the sound grows along with deviation of the wave vector from the axis of the order parameter. The sound speed is maximal for orthogonal propagation.

Immediately below the critical temperature $T_{c}$ the velocity of zero sound decreases when the temperature goes down, and the undamped wave disappears at some temperature $T_{1}\left(\theta_{\mathbf{q}}\right)<T_{c}$ when the sound velocity becomes smaller than Fermi velocity. (although the mode with exponentially small damping can exist in some region below this temperature). Thus the undamped collective excitation may or may not exist at some temperature, depending on the values of $F_{0}$ and $F_{1}$. If it does, its velocity will always be greater than Fermi velocity.

In our analysis, we have assumed that the axis of the order parameter is equally oriented everywhere. It is necessary to notice, however, that texture effects can orient different parts of the sample differently, and therefore give a range of frequency shifts which together appear as a broad line. The texture effects can be minimized by an external magnetic field, although the magnetic field has no consequence on the dispersion of the sound wave except that it serves to fix the relative orientation of the spin-orbital wave function associated with the order parameter, if the dipole interaction is taken into account.

On the other hand the texture effects could play an important role in neutrino cooling of neutron star at the latest stage. Indeed, the sound wave can emit a neutrino pair through neutral weak currents while crossing the border where the axis changes its direction. Neutrino radiation is possible also due to collisions of sound waves [13]. As already mentioned in the Introduction, the sound waves are known to play an important role also in a superfluid heat conduction when the transverse electron motion is strongly suppressed by a magnetic field [12]. Various applications of the results obtained in this paper will be considered elsewhere.

All the results of this paper depend on the Fermi-liquid functions $F_{0}, F_{1}$ which parametrize the normal Fermi liquid. The only way to evaluate these functions for a superdense asymmetric nuclear matter is to estimate their values from first-principles calculations. Although, 
in practice, such work is in progress [29 32], the complete information on the Landau parameters for a neutron matter is not available at the moment. Only the well-known conditions [17] of the matter stability with respect to long-wave perturbations can be used in order to limit the Landau parameters.

[1] R. Tamagaki, Prog. Theor. Phys. 44, 905 (1970).

[2] M. Hoffberg, A. E. Glassgold, R. W. Richardson and M. Ruderman, Phys. Rev. Letters 24, 775 (1970).

[3] T. Takatsuka, Prog. Theor. Phys. 48, 1517 (1972).

[4] M. Baldo, J. Cugnon, A. Lejeune and U. Lombardo, Nucl. Phys. A 536, 349 (1992).

[5] Ø. Elgarøy, L. Engvik, M. Hjorth-Jensen, E. Osnes, Nucl. Phys. A 607, 425 (1996).

[6] V. V. Khodel, V. A. Khodel, and J. W. Clark, Nucl. Phys. A 679, 827 (2001).

[7] D. J. Dean and M. Hjorth-Hensen, Rev. Mod. Phys. 75, 607 (2003).

[8] M.V. Zverev, J. W. Clark, and V. A. Khodel, Nucl. Phys. A 720, 20 (2003).

[9] L. B. Leinson, Phys. Rev. C 81, 025501 (2010).

[10] L. B. Leinson, Phys. Lett. B 689, 60 (2010).

[11] L. B. Leinson, Phys. Rev. C 82, 065503 (2010).

[12] D. N. Aguilera, V. Cirigliano, J. A. Pons, S. Reddy, and R. Sharma, Phys. Rev. Lett. 102, 091101 (2009).

[13] P. F. Bedaque, G. Rupak, and M. J. Savage, Phys. Rev. C 68, 065802 (2003).

[14] Y. Nambu, Phys. Rev. 117, 648 (1960).

[15] L. B.Leinson and A. Pérez, Phys. Lett. B 638, 114 (2006).

[16] L. B. Leinson, Nucl. Phys. A 687, 489 (2001).

[17] A. B. Migdal, Theory of Finite Fermi Systems and Applications to Atomic Nuclei (Interscience, London, 1967).

[18] M. E. Gusakov, Phys. Rev. C 81, 025804 (2010).

[19] A. J. Leggett, Phys. Rev. 147, 119 (1966).

[20] P. Wölfe, Phys. Rev. Lett. 30, 1169 (1973).

[21] P. Wölfe, Phys. Rev. Lett. 31, 1437 (1973).

[22] H. Ebisawa and K. Maki, Prog. Theor. Phys. 51, 337 (1974). 
[23] L. B. Leinson, Phys. Rev. C 78, 015502 (2008).

[24] A. J. Leggett, Rev. Mod. Phys. 47, 331 (1975).

[25] A. J. Leggett, Phys. Rev. 140, A1869 (1965).

[26] D. Pines and P. Nozières, Theory of Quantum Liquids (Benjamin, New York, 1966).

[27] A. I. Larkin and A. B. Migdal, Zh. Experim. i Teor. Fiz. 44, 1703 (1963) [Sov. Phys. JETP $17,1146(1963)]$.

[28] J. Schrieffer, Theory of Superconductivity (W. Benjamin, New York, 1964), p. 157.

[29] J. Lehr, M. Effenberger, H. Lenske, S. Leupold and U. Mosel, Phys. Lett. B483, 324 (2000).

[30] F. Frömel, H. Lenske, U. Mosel, Nucl. Phys. A723, 544 (2003).

[31] E. N. E. van Dalen, C. Fuchs, and A. Faessler, Phys. Rev. Lett. 95, 022302 (2005).

[32] P. Konrad, H. Lenske, U. Mosel, Nucl.Phys.A 756, 192 (2005). 